\title{
Numerical Investigation of the Role of Turbulent Bursting in Sediment Entrainment
}

\author{
Fu-Chun $\mathrm{Wu}^{1}$ and Men-Rong Jiang ${ }^{2}$
}

\begin{abstract}
In this work we incorporate a Gram-Charlier-type joint probability distribution of near-bed two-dimensional instantaneous velocities into a simple mechanistic model to investigate the role of turbulent bursting in sediment entrainment. The results reveal that under typical values of bed-shear stress (>3 Pa), the time fractions of Quadrants 1-4 (Q1-Q4) remain constantly as 16, 34, 19, and 31\%, respectively. Entrainment of the fine sediment mixtures is dominated by the lifting mode, whereas entrainment of the coarse ones is dominated by rolling. Sweeps (Q4) are consistently the most significant contributor to entrainment under various types of sediment mixtures. As the standard deviation of grain-size distribution increases, the hiding effect exerted on the finer grains of the mixture is reduced, leading to the elevated correction factors for effective hydrodynamic forces, and thus the reduced threshold velocities for entrainment. The reduced thresholds would, in turn, enhance the fractional contributions of ejections and inward interactions (Q2 and Q3), which are associated with negative longitudinal velocity fluctuations, such that the fractional contribution of outward interactions (Q1) would become less significant.
\end{abstract}

DOI: 10.1061/(ASCE)0733-9429(2007)133:3(329)

CE Database subject headings: Sediment; Entrainment; Velocity; Shear stress.

\section{Introduction}

Turbulent bursting constitutes a near-bed coherent structure in open-channel flows (Nezu and Nakagawa 1993). Burst events can be divided into four quadrants [Quadrants 1-4 (Q1-Q4)] by their longitudinal and vertical velocity fluctuations $u^{\prime}$ and $v^{\prime}$, namely, outward interactions $\left(u^{\prime}>0, v^{\prime}>0\right)$, ejections $\left(u^{\prime}<0, v^{\prime}>0\right)$, inward interactions $\left(u^{\prime}<0, v^{\prime}<0\right)$, and sweeps $\left(u^{\prime}>0, v^{\prime}<0\right)$. A better understanding of the coupling between near-bed turbulence and sediment entrainment would facilitate prediction of bed-load transport in fluvial morphodynamic modeling. Limited by the difficulties inherent in simultaneous measurements of local sediment movement and adjacent fluid velocities at turbulenceresolving frequencies, accurate measurements of the interactions between turbulent bursting and sediment motion were first made possible with a synchronized laser-Doppler velocimetry (LDV) and high-speed cinematography (Nelson et al. 1995). From the measurement results, Nelson et al. (1995) found that sweeps (Q4) collectively move the majority of the sediment; outward interactions (Q1) individually move as much sediment as sweeps; and ejections and inward interactions (Q2 and Q3) move much less

${ }^{1}$ Professor, Dept. of Bioenvironmental Systems Engineering, Hydrotech Research Institute and Center for Ecological Engineering, National Taiwan Univ., Taipei 106, Taiwan, R.O.C. E-mail: fcwu@ ntu.edu.tw

${ }^{2}$ Graduate Research Assistant, Dept. of Bioenvironmental Systems Engineering, National Taiwan Univ., Taipei 106, Taiwan, R.O.C.

Note. Discussion open until August 1, 2007. Separate discussions must be submitted for individual papers. To extend the closing date by one month, a written request must be filed with the ASCE Managing Editor. The manuscript for this technical note was submitted for review and possible publication on May 3, 2005; approved on August 28, 2006. This technical note is part of the Journal of Hydraulic Engineering, Vol. 133, No. 3, March 1, 2007. (CASCE, ISSN 0733-9429/2007/3-329-334/ $\$ 25.00$. sediment than sweeps and outward interactions. However, the observations were made under very limited conditions of flow and sediment, thus the generality of their findings remains to be further investigated.

In this work we incorporate a Gram-Charlier (GC) joint probability distribution of near-bed two-dimensional (2D) instantaneous velocities into a simple mechanistic model to replicate the burst events and predict the incipient motions of sediment. The role of turbulent bursting in sediment entrainment under various flow strengths and grain size distributions are explored numerically.

\section{Probability Distribution of Near-Bed Velocities}

Several previous studies (e.g., Frenkiel and Klebanoff 1973; Nakagawa and Nezu 1977; Wu and Yang 2004) have suggested that the GC probability density function (pdf) well describes the distribution of near-bed velocity fluctuations. The third-order GC joint pdf $g_{3}(U, V)$ is used in this work (Fig. 1), which can be expressed as

$$
g_{3}(U, V)=g_{0}(U, V) \cdot\left(1+L_{1}+L_{2}+L_{3}+L_{4}\right)
$$

where

$$
\begin{gathered}
g_{0}(U, V)=\frac{1}{2 \pi \sqrt{1-R_{u v}^{2}}} \exp \left[-\frac{U^{2}-2 R_{u v} U V+V^{2}}{2\left(1-R_{u v}^{2}\right)}\right] \\
L_{1}=\frac{S_{u}}{3 !}\left[-\left(\frac{R_{v}}{1-R_{u v}^{2}}\right)^{3}+\frac{3 R_{v}}{\left(1-R_{u v}\right)^{2}}\right] \\
L_{2}=\frac{M_{21}}{2 !}\left[\frac{3 R_{u v} U-2 R_{u v}^{2} V-V}{\left(1-R_{u v}^{2}\right)^{2}}-\frac{R_{u} R_{v}^{2}}{\left(1-R_{u v}^{2}\right)^{3}}\right]
\end{gathered}
$$




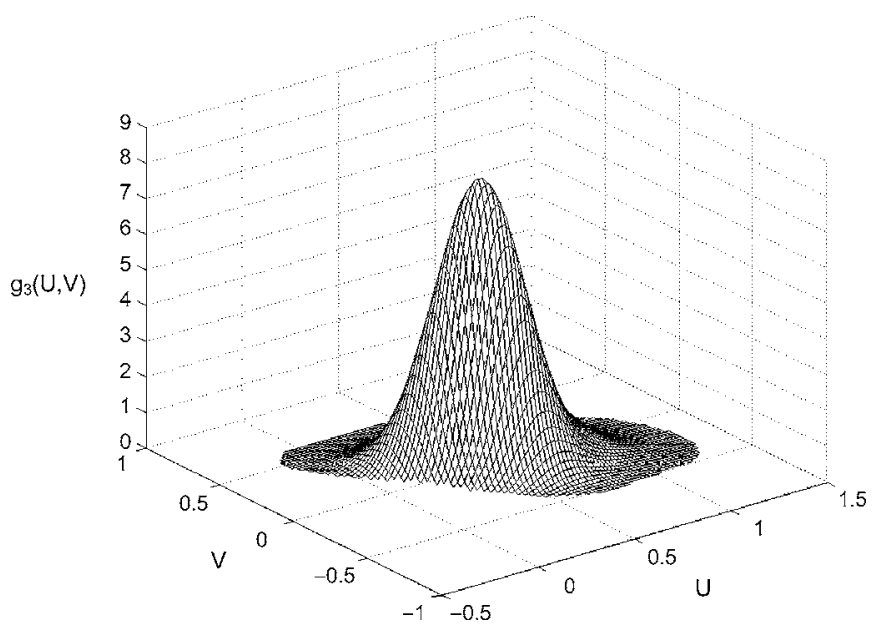

Fig. 1. Three-dimensional plot showing the third-order Gram-Charlier joint pdf of the normalized velocity fluctuations $\left(u_{*}=0.055 \mathrm{~m} / \mathrm{s}\right)$

$$
\begin{gathered}
L_{3}=\frac{M_{12}}{2 !}\left[\frac{3 R_{u v} V-2 R_{u v}^{2} U-U}{\left(1-R_{u v}^{2}\right)^{2}}-\frac{R_{v} R_{u}^{2}}{\left(1-R_{u v}^{2}\right)^{3}}\right] \\
L_{4}=\frac{S_{v}}{3 !}\left[-\left(\frac{R_{u}}{1-R_{u v}^{2}}\right)^{3}+\frac{3 R_{u}}{\left(1-R_{u v}\right)^{2}}\right]
\end{gathered}
$$

in which $U=u^{\prime} / \sigma_{u} ; V=v^{\prime} / \sigma_{v} ; u^{\prime}=u_{b}-\bar{u}_{b} ; v^{\prime}=v_{b}-\bar{v}_{b}, u_{b}$ and $v_{b}=$ near-bed instantaneous longitudinal and vertical velocities; $\bar{u}_{b}$ and $\bar{v}_{b}=$ time-averaged velocities; $\sigma_{u}$ and $\sigma_{v}=$ standard deviations; $R_{u v}=\left\langle u^{\prime} v^{\prime}\right\rangle / \sigma_{u} \sigma_{v}=$ correlation coefficient, where \langle\rangle denotes the ensemble mean; $R_{u}=R_{u v} V-U ; R_{v}=R_{u v} U-V ; S_{u}=\left\langle u^{\prime 3}\right\rangle / \sigma_{u}^{3}$; and $S_{v}=\left\langle v^{\prime 3}\right\rangle / \sigma_{v}^{3}=$ skewness factors; and $M_{21}=\left\langle u^{\prime 2} v^{\prime}\right\rangle / \sigma_{u}^{2} \sigma_{v}$ and $M_{12}=\left\langle u^{\prime} v^{\prime 2}\right\rangle / \sigma_{u} \sigma_{v}^{2}=$ diffusion factors. The values of these parameters are determined as follows.

The near-bed correlation coefficient $R_{u v}$ typically ranges from -0.4 to -0.5 (Pope 2000). Given a compilation of data for various roughness regimes and experimental settings $(\mathrm{Wu}$ and Yang 2004), we adopt a value of $R_{u v}=-0.45$ throughout this study. Based on such data, Wu and Yang (2004) proposed $\sigma_{u} / u_{*}=-0.187 \ln \left(k_{s}^{+}\right)+2.93$ and $S_{u}=0.102 \ln \left(k_{s}^{+}\right)$for smooth and transitional regimes $\left(k_{s}^{+} \leq 70\right)$, where $u_{*}=\sqrt{\tau_{0} / \rho} ; \tau_{0}=$ bed-shear stress; $\rho=$ density of fluid; $k_{s}^{+}=u * k_{s} / \nu ; k_{s}=2 D_{50} ; D_{50}=$ median grain size; and $\nu=$ kinematic viscosity of fluid. For rough regimes $\left(k_{s}^{+}>70\right)$, constant values of $\sigma_{u} / u_{*}=2.14$ and $S_{u}=0.43$ were suggested. In addition, constant values of $\sigma_{v} / u_{*}=1, S_{v}=0, M_{12}=0.1$, and $M_{21}=-0.04$ are adopted in this work because no significant variations of these parameters are observed from the compiled data set.

It has been pointed out that the GC pdf may result in small negative values at tail regions (Frenkiel and Klebanoff 1973). Although, in principle, negative densities are unacceptable, the resulting probability distribution is usable if appropriate precautions are taken by specifying zero densities to tail regions and rescaling the density function (generally, $\leq 1 \pm 0.05 \%$ ) such that the cumulative probability (integration of pdf over $U$ and $V$ ) sums up to unity. The instantaneous velocities $u_{b}$ and $v_{b}$ can be decomposed into a mean velocity and a fluctuation component, where the longitudinal mean velocity $\bar{u}_{b}$ is evaluated using the approach detailed in Wu and Yang (2004), and the vertical mean velocity $\bar{v}_{b}$ is taken to be zero.

\section{Mechanistic Model for Sediment Entrainment}

A simple mechanistic model presented by Wu and Yang (2004) is adopted in this work. Only the conceptual framework is outlined here; the readers are referred to the original work for details. The external forces applied on a resting sediment particle include the submerged weight $W$ and instantaneous hydrodynamic forces, which can be resolved into an effective drag $F_{D}$ and an effective lift $F_{L}$, both formulated in terms of $u_{b}$, as adopted by Cheng and Chiew (1999), Fischer et al. (2002), and Wu and Yang (2004), i.e.

$$
F_{D}=\xi_{i} C_{D} \frac{\rho A u_{b}^{2}}{2}, \quad F_{L}=\xi_{i} C_{L} \frac{\rho A u_{b}^{2}}{2}
$$

where $\xi_{i}=$ hiding-exposure correction factor for grain size $D_{i} ; C_{D}$ and $C_{L}=\mathrm{drag}$ and lift coefficients, respectively; and $A=$ exposed frontal area. The approach adopted herein implies that the instantaneous lift is in phase with the instantaneous drag (since both are evaluated using $u_{b}$ ), which at best represents a necessarily simplified treatment of force balance because using $v_{b}$ to evaluate the instantaneous lift lacks any physical evidence. Although it has been also argued that in turbulent boundary layers the Reynolds stress $\left(-\rho u^{\prime} v^{\prime}\right)$ plays a significant role in producing shear forces, an increasing number of studies indicated that the near-bed longitudinal velocity, rather than the Reynolds stress, is most responsible for sediment motions (e.g., Williams et al. 1989; Nelson et al. 1995; Schmeeckle and Nelson 2003). In this work, the 2D effect of turbulent bursting is nevertheless accounted for through the GC joint pdf, as described in the next section.

The criterion for a static particle to be instantaneously entrained in the rolling mode can be expressed by

$$
F_{D} L_{D}+F_{L} L_{L}>W L_{W}
$$

where $L_{D}, L_{L}$, and $L_{W}=$ moment arms of $F_{D}, F_{L}$, and $W$, respectively. Substituting the expressions of the forces and moment arms into Eq. (3) leads to

$$
u_{b}^{2}>B_{R}^{2}
$$

where $B_{R}=$ threshold velocity for incipient rolling. Similarly, the criterion for a static particle to be instantaneously entrained in the lifting mode can be expressed by

$$
F_{L}>W
$$

Substituting the expressions of the forces into Eq. (5) yields

$$
u_{b}^{2}>B_{L}^{2}
$$

where $B_{L}=$ threshold velocity for incipient lifting. It has been shown that the lifting threshold $B_{L}$ is higher than the rolling threshold $B_{R}$ (Wu and Chou 2003).

\section{Probabilities of Sediment Entrainment}

The probability of sediment entrainment in the rolling mode is defined by

$$
P_{R}=P\left[\left(B_{R}^{2}<u_{b}^{2}<B_{L}^{2}\right) \cap\left(u_{l} \leq u_{b} \leq u_{u}\right) \mid\left(v_{l} \leq v_{b} \leq v_{u}\right)\right]
$$

where the instantaneous velocities $u_{l}=\bar{u}_{b}+u_{l}^{\prime}$ and $u_{u}=\bar{u}_{b}+u_{u}^{\prime}$, and $u_{l}^{\prime}$ and $u_{u}^{\prime}=$ lower and upper bounds of $u^{\prime}$ corresponding to the region within which $g_{3}(U, V)$ is positive (Fig. 1); and $v_{l}=\bar{v}_{b}+v_{l}^{\prime}$ and $v_{u}=\bar{v}_{b}+v_{u}^{\prime}$, and $v_{l}^{\prime}$ and $v_{u}^{\prime}=$ lower and upper bounds of $v^{\prime}$ corresponding to the region with positive $g_{3}(U, V)$. The conditional probability defined by Eq. (7) implies that for each $v^{\prime}$ be- 
tween $\left(v_{l}^{\prime}, v_{u}^{\prime}\right)$ there exists a corresponding range of $u^{\prime}$ between $\left(u_{l}^{\prime}, u_{u}^{\prime}\right)$ through which the $2 \mathrm{D}$ constraint of turbulent bursting is imposed. The rolling probability $P_{R}$ is then evaluated by integrating $g_{3}(U, V)$ over suitable ranges of $U$ and $V$, i.e.

$$
P_{R}=\int_{V_{R}} \int_{U_{R}} g_{3}(U, V) d U d V
$$

where $U_{R}$ and $V_{R}$ denote the ranges of $U$ and $V$ that simultaneously meet the constraints for incipient rolling and joint pdf of turbulent bursting. The mean rolling probability, PR, is the expected value of Eq. (8) over the full ranges of the supporting grain size $D_{k}$, exposure height $\Delta_{j}$, and friction height $\Delta_{k}$ (for details see $\mathrm{Wu}$ and Yang 2004).

Similarly, the probability of sediment entrainment in the lifting mode is defined by

$$
P_{L}=P\left[\left(B_{L}^{2}<u_{b}^{2}\right) \cap\left(u_{l} \leq u_{b} \leq u_{u}\right) \mid\left(v_{l} \leq v_{b} \leq v_{u}\right)\right]
$$

The lifting probability $P_{L}$ is evaluated by integrating $g_{3}(U, V)$ over suitable ranges of $U$ and $V$, i.e.

$$
P_{L}=\int_{V_{L}} \int_{U_{L}} g_{3}(U, V) d U d V
$$

where $U_{L}$ and $V_{L}$ denote the ranges of $U$ and $V$ that simultaneously meet the constraints for incipient lifting and joint pdf of turbulent bursting. The mean lifting probability, PL, is the expected value of Eq. (10) over the full ranges of $\Delta_{j}$ and $\Delta_{k}$. Since rolling and lifting are two independent modes, the total entrainment probability $\mathrm{PT}$ is given by $\mathrm{PR}+\mathrm{PL}$.

\section{Quadrant Analyses}

The proportion of time occupied by the burst events in the $i$ th quadrant, denoted as $T_{i}$, is evaluated by

$$
T_{i}=\int_{V_{i}} \int_{U_{i}} g_{3}(U, V) d U d V \quad \text { for } i=1, \ldots, 4
$$

where $U_{i}$ and $V_{i}=$ ranges of integration in the $i$ th quadrant. The fractional contribution (FC) of the $i$ th quadrant to sediment entrainment, denoted as $\mathrm{FC}_{i}$, is evaluated by

$$
\mathrm{FC}_{i}=\left(\mathrm{PR}_{i}+\mathrm{PL}_{i}\right) / \mathrm{PT} \quad \text { for } i=1, \ldots, 4
$$

where $\mathrm{PR}_{i}=$ expected value of $P_{R, i}$ over the full ranges of $\left(D_{k}, \Delta_{j}\right.$, $\left.\Delta_{k}\right)$; and $\mathrm{PL}_{i}=$ expected value of $P_{L, i}$ over the full ranges of $\left(\Delta_{j}\right.$, $\left.\Delta_{k}\right)$, in which $P_{R, i}=\int_{V_{R, i}} \int_{U_{R, i}} g_{3}(U, V) d U d V, \quad P_{L, i}=\int_{V_{L, i}} \int_{U_{L, i}} g_{3}$ $(U, V) d U d V ; U_{R, i}$ and $V_{R, i}=$ partial ranges of $U_{R}$ and $V_{R}$ in the $i$ th quadrant; and $U_{L, i}$ and $V_{L, i}=$ partial ranges of $U_{L}$ and $V_{L}$ in the $i$ th quadrant.

\section{Results Verification}

\section{Time Fractions of Four Quadrants}

The predicted proportions of time occupied by four quadrants are verified with two sets of experimental data. The first was collected by Nelson et al. (1995) in a recirculating flume with a well-sorted sand bed. The grain size was lognormally distributed with a mean of $0.9 \mathrm{~mm}$ and a standard deviation of $0.1 \mathrm{~mm}$. Measurements of the fluid velocities were made by the LDV at a point $5 \mathrm{~mm}$ above the bed. Mean flow conditions were $\tau_{0}=0.78 \mathrm{~Pa}$,

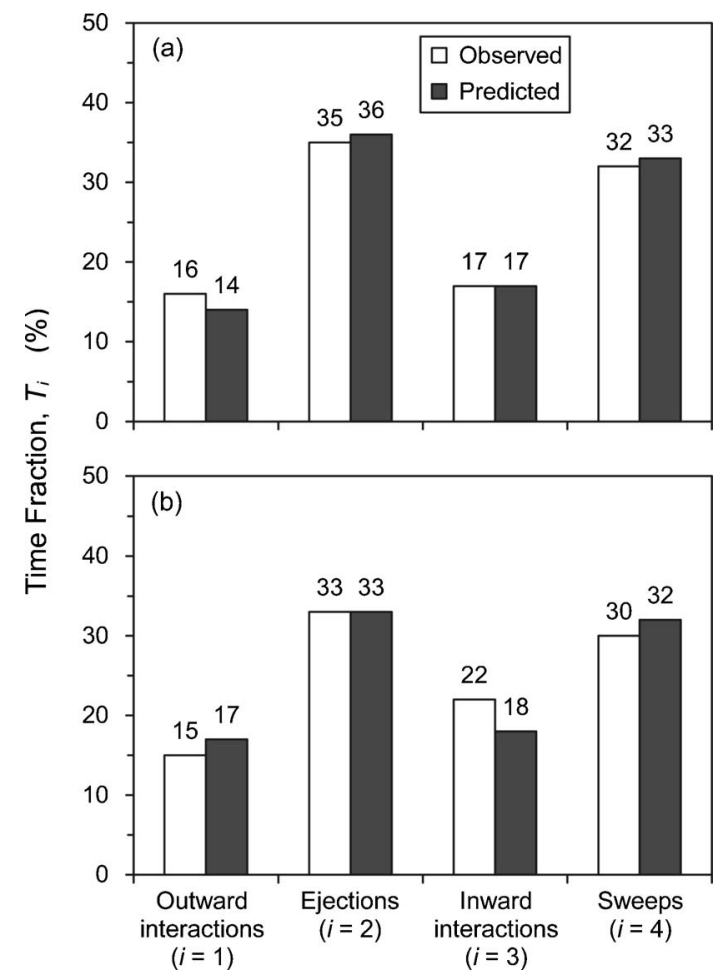

Fig. 2. Time fractions of four quadrants for (a) $\tau_{0}=0.78 \mathrm{~Pa}$, mean grain size $=0.9 \mathrm{~mm}$; (b) $\tau_{0}=8.7 \mathrm{~Pa}$, uniform grain size $=8 \mathrm{~mm}$

$\bar{u}_{b}=32.9 \mathrm{~cm} / \mathrm{s}, \quad \sigma_{u}=5.3 \mathrm{~cm} / \mathrm{s}, \quad \sigma_{v}=3.2 \mathrm{~cm} / \mathrm{s}$, and $R_{u v}=-0.44$, classified as a transitional regime $\left(k_{s}^{+} \cong 50\right)$. The predicted and observed patterns of $T_{i}$ are shown in Fig. 2(a), where satisfactory agreement is demonstrated. Ejections (Q2) were the most common events; sweeps (Q4) were slightly less common; these two types of burst events occupied more than $65 \%$ of the total duration. Inward interactions (Q3) were slightly more common than outward interactions (Q1).

The second set of data was collected by Papanicolaou et al. (2001) using a flume with lead spherical particles $(8 \mathrm{~mm}$ in diameter) placed atop a bed packed with glass beads of $8 \mathrm{~mm}$ in size. Three packing densities of lead spheres were used to simulate the isolated, wake-interference, and skimming flow regimes. Only the data for the skimming flow regime (70\% packing density) are adopted here because the other two apparently represent different bed configurations from the densely packed bed considered in this study. Velocity measurements were made by the LDV at a point $4.7 \mathrm{~mm}$ above the lead sphere. Flow conditions were $\tau_{0}=8.7 \mathrm{~Pa}, \bar{u}_{b}=34.6 \mathrm{~cm} / \mathrm{s}, \sigma_{u}=16.1 \mathrm{~cm} / \mathrm{s}$, and $\sigma_{v}=10.5 \mathrm{~cm} / \mathrm{s}$, classified as a rough regime $\left(k_{s}^{+} \cong 1,500\right)$. The predicted and observed patterns of $T_{i}$ are shown in Fig. 2(b), where satisfactory agreement is again demonstrated. Similar to the results shown in Fig. 2(a), ejections and sweeps (Q2 and Q4) were the most common events, with the time fractions of both slightly reduced, which was probably due to the $70 \%$ packing density. Although the flow regimes used in the above two experiments were different (transitional versus rough regimes), the patterns of $T_{i}$ shown in Figs. 2( $\mathrm{a}$ and $\mathrm{b}$ ) were quite similar, implying that the time fractions of four quadrants may not be very sensitive to flow regimes. We will address this issue in the section of model applications. 

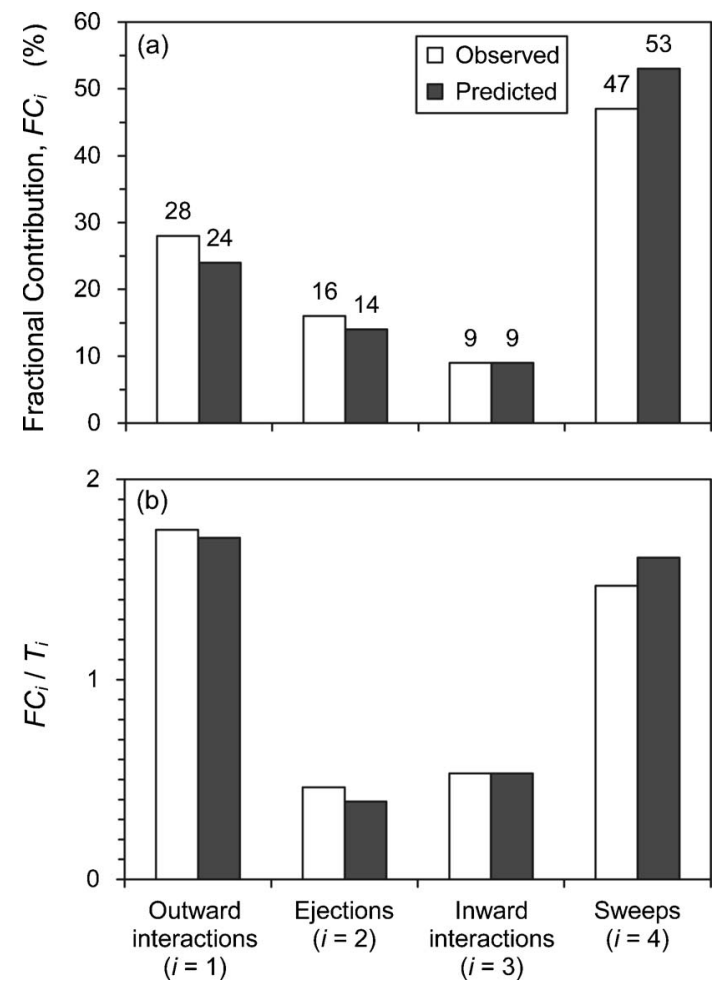

Fig. 3. (a) Fractional contributions; (b) unit fractional contributions of four quadrants for $\tau_{0}=0.78 \mathrm{~Pa}$ and mean grain size $=0.9 \mathrm{~mm}$

\section{Fractional Contributions to Sediment Entrainment}

The simultaneous measurements of fluid velocities and sediment movement performed by Nelson et al. (1995) using a synchronized LDV and high-speed CCD are used here to verify the predicted fractional contributions of four quadrants to sediment entrainment. The predicted and observed patterns of $\mathrm{FC}_{i}$ are shown in Fig. 3(a), where satisfactory agreement is demonstrated. Sweeps (Q4) were the major contributor to sediment entrainment, with $\sim 50 \%$ of the entrainment being attributed to these events; outward interactions (Q1) contributed about 1/2 of that amount, roughly equal to the combined contribution of ejections $(\mathrm{Q} 2)$ and inward interactions (Q3). Since both sweeps (Q4) and outward interactions $(\mathrm{Q} 1)$ are associated with positive $u^{\prime}$, the significant contributions of these two quadrants may be attributed to the greater longitudinal velocities. Nelson et al. (1995) further argued that the fractional contribution of sweeps (Q4) was more significant than that of outward interactions (Q1) because sweeps were much more common events [Fig. 2(a)], which is confirmed by the patterns of $\mathrm{FC}_{i} / T_{i}$ shown in Fig. 3(b). It is revealed that Q1 and Q4 were those two quadrants with the highest values of $\mathrm{FC}_{i} / T_{i}$, indicating that outward interactions (Q1) individually entrained as much sediment as sweeps (Q4). Generality of this argument will be investigated in the following section.

\section{Model Applications}

In this section, we investigate the effect of flow strength on the time fractions of four quadrants. The proposed model is also applied to explore the effect of grain-size distribution on the fractional contributions of four quadrants to sediment entrainment.

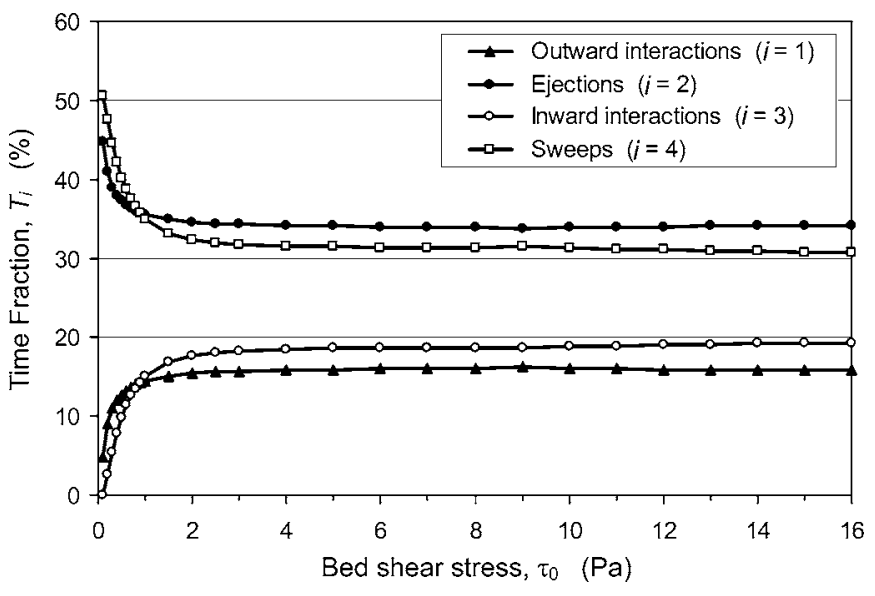

Fig. 4. Variations of time fraction with bed-shear stress (each data point represents the mean value corresponding to a range of $\theta$ between 0.01 and 10 )

\section{Effect of Flow Strength on Time Fractions}

To investigate the effects of flow strength $\left(\tau_{0}\right)$ and grain roughness $\left(D_{50}\right)$ on the time fractions of four quadrants, we calculate the values of $T_{i}$ for a number of $\tau_{0}$ ranging from 0.1 to $16 \mathrm{~Pa}$. For each $\tau_{0}$, the grain sizes corresponding to a typical range of $\theta$ (Shields number) between 0.01 and 10 are evaluated, and the resulting values of $k_{s}^{+}$and $T_{i}$ are then determined. From the results we find that for each $\tau_{0}$, the values of $T_{i}$ exhibit little variations although the grain size and $k_{s}^{+}$are increased by three orders of magnitude, indicating that grain roughness has negligible effects on the time fractions of four quadrants. Shown in Fig. 4 are the relations between $T_{i}$ and $\tau_{0}$, where significant variations of $T_{i}$ are observed only in a narrow range of $\tau_{0}$ between 0 and $3 \mathrm{~Pa}$. Variations of $T_{i}$ are, however, not apparent for $\tau_{0}>3 \mathrm{~Pa}$, where the values of $T_{1}-T_{4}$ remain as $16,34,19$, and $31 \%$, respectively. With such results in view, it is not surprising that the patterns of $T_{i}$ shown in Figs. 2(a and b) are so similar. Fig. 4 also reveals that sweeps (Q4) and ejections (Q2) would become extremely common at very low values of $\tau_{0}$, which remains open to further investigations. LDV measurements are currently undertaken by the writers to verify such results.

\section{Effect of Grain Size Distribution on Fractional Contributions}

Given that the time fractions of four quadrants are affected neither by grain roughness nor the values of $\tau_{0}$ typical of open-channel flows, we speculate that the fractional contributions of four quadrants to sediment entrainment would respond to the variation of grain-size distribution. To explore the effect of grain-size distribution, two types of sediment mixture (fine and coarse) are used here. Both mixtures have lognormally distributed grain sizes, which cover a range from 0.13 to $9.77 \mathrm{~mm}$ for the fine mixture $\left(D_{50}=1 \mathrm{~mm}\right)$, and from 0.47 to $52.5 \mathrm{~mm}$ for the coarse mixture $\left(D_{50}=6 \mathrm{~mm}\right)$. For each type of mixture, two degrees of grain sorting are considered, namely, well sorted $\left(\sigma_{g}=2\right)$ and poorly sorted $\left(\sigma_{g}=3.6\right)$, where $\sigma_{g}=$ geometric standard deviation $=D_{84} / D_{50}$. The predicted fractional contributions associated with various sediment mixtures are shown in Table 1 , where the results associated with uniform sediments $\left(\sigma_{g}=1\right)$ are also demonstrated to provide a basis for comparisons. The values of $\tau_{0}$ used for the 
Table 1. Fractional Contributions of Four Quadrants to Entrainment of Various Sediment Mixtures (Units in \%)

\begin{tabular}{|c|c|c|c|c|c|}
\hline \multirow[b]{2}{*}{$\begin{array}{l}\text { Degree } \\
\text { of grain } \\
\text { sorting }\end{array}$} & $\mathrm{FC}_{1}$ & $\mathrm{FC}_{2}$ & $\mathrm{FC}_{3}$ & $\mathrm{FC}_{4}$ & \multirow[b]{2}{*}{$\begin{array}{l}\sum \mathrm{PL}_{i} / \mathrm{PT} \\
\sum \mathrm{PR}_{i} / \mathrm{PT}\end{array}$} \\
\hline & $\begin{array}{l}\mathrm{PL}_{1} / \mathrm{PT} \\
\mathrm{PR}_{1} / \mathrm{PT}\end{array}$ & $\begin{array}{l}\mathrm{PL}_{2} / \mathrm{PT} \\
\mathrm{PR}_{2} / \mathrm{PT}\end{array}$ & $\begin{array}{l}\mathrm{PL}_{3} / \mathrm{PT} \\
\mathrm{PR}_{3} / \mathrm{PT}\end{array}$ & $\begin{array}{l}\mathrm{PL}_{4} / \mathrm{PT} \\
\mathrm{PR}_{4} / \mathrm{PT}\end{array}$ & \\
\hline \multicolumn{6}{|c|}{ (a) Fine sediment mixture $\left(D_{50}=1 \mathrm{~mm}\right)$} \\
\hline \multirow{3}{*}{$\begin{array}{l}\text { Uniform } \\
\left(\sigma_{g}=1\right)\end{array}$} & 22.0 & 18.1 & 13.5 & 46.5 & - \\
\hline & 16.9 & 6.9 & 6.4 & 37.1 & 67.2 \\
\hline & 5.1 & 11.2 & 7.1 & 9.4 & 32.8 \\
\hline \multirow{3}{*}{$\begin{array}{l}\text { Well sorted } \\
\left(\sigma_{g}=2\right)\end{array}$} & 21.6 & 18.7 & 14.2 & 45.5 & - \\
\hline & 16.6 & 4.6 & 4.5 & 36.1 & 61.8 \\
\hline & 5.0 & 14.1 & 9.7 & 9.4 & 38.2 \\
\hline \multirow{3}{*}{$\begin{array}{l}\text { Poorly sorted } \\
\left(\sigma_{g}=3.6\right)\end{array}$} & 17.6 & 28.3 & 17.7 & 36.4 & - \\
\hline & 15.5 & 17.8 & 12.6 & 32.3 & 78.2 \\
\hline & 2.1 & 10.5 & 5.1 & 4.1 & 21.8 \\
\hline
\end{tabular}

\begin{tabular}{lrccrr}
\hline \multicolumn{5}{c}{ (b) Coarse sediment mixture $\left(D_{50}=6 \mathrm{~mm}\right)$} \\
\hline $\begin{array}{l}\text { Uniform } \\
\left(\sigma_{g}=1\right)\end{array}$ & 14.9 & 25.0 & 10.8 & 49.3 & - \\
& 0.3 & 0 & 0 & 3.6 & 3.9 \\
& 14.6 & 25.0 & 10.8 & 45.7 & 96.1 \\
& & & & & \\
Well sorted & 16.1 & 29.8 & 6.8 & 47.3 & - \\
$\left(\sigma_{g}=2\right)$ & 1.4 & 1.2 & 0 & 6.6 & 9.2 \\
& 14.7 & 28.6 & 6.8 & 40.7 & 90.8 \\
& 16.4 & 33.2 & 9.6 & 40.8 & - \\
$\begin{array}{l}\text { Poorly sorted } \\
\left(\sigma_{g}=3.6\right)\end{array}$ & 4.6 & 8.4 & 1.3 & 13.2 & 27.5 \\
& 11.8 & 24.8 & 8.3 & 27.6 & 72.5 \\
\hline
\end{tabular}

fine and coarse sediment mixtures are 3 and $10 \mathrm{~Pa}$, respectively, with the corresponding values of $k_{s}^{+}=110$ and 1,200 , both classified as fully rough regimes.

Entrainment of the fine sediment mixtures is dominated by lifting, with consistently more than $60 \%$ entrained in the lifting mode (respectively, 67, 62, and 78\% for the uniform, well-, and poorly sorted sediments); whereas entrainment of the coarse sediment mixtures is dominated by rolling, with consistently less than $30 \%$ entrained in the lifting mode (respectively, 4, 9, and $28 \%$ for the uniform, well-, and poorly sorted sediments). The fractional contributions of sweeps (Q4) are consistently the most significant ones under various sediment mixtures, because of both the greater longitudinal velocities $\left(u^{\prime}>0\right)$ and time fractions $\left(T_{4}=31 \%\right)$. Outward interactions (Q1) are the second significant contributor only under the uniform and well-sorted fine sediments. Under the coarse sediment mixtures and the poorly sorted fine sediment, however, the fractional contributions of ejections (Q2) exceed the corresponding values of outward interactions (Q1). In other words, the earlier argument made by Nelson et al. (1995), claiming that sweeps $(\mathrm{Q} 4)$ and outward interactions $(\mathrm{Q} 1)$ are two major contributors to entrainment, seems not to be valid for all types of sediment mixtures with different degrees of sorting.
To further look into this problem, the dominant mode of entrainment in each quadrant is individually identified by the values of $\mathrm{PL}_{i} / \mathrm{PT}$ and $\mathrm{PR}_{i} / \mathrm{PT}$ listed in Table 1 . Under the uniform and well-sorted fine sediments for which outward interactions (Q1) are the second major contributor, the dominant mode of entrainment in Q1 and Q4 is lifting, different from the rolling mode that dominates the sediment entrainment in Q2 and Q3. However, under the coarse sediment mixtures and the poorly sorted fine sediment for which ejections (Q2) are the second major contributor, the dominant mode of entrainment in four quadrants is consistent, which is lifting under the poorly sorted fine sediment but is rolling under the coarse sediment mixtures. Therefore, whether outward interactions $(\mathrm{Q} 1)$ or ejections $(\mathrm{Q} 2)$ are the second major contributor depends on if the dominant mode of entrainment in Q1 and Q4 (both with $u^{\prime}>0$ ) is consistent with that in Q2 and Q3 (both with $u^{\prime}<0$ ), which is elucidated below in more detail.

In Fig. 4 we see that outward interactions (Q1) are very rare events, with the time fraction $T_{1}$ consistently no more than $16 \%$. As such, their remarkable contribution to entrainment relies on the greater longitudinal velocities $\left(u^{\prime}>0\right)$ but not the time occupied. Under the uniform fine sediment, lifting is the dominant mode of entrainment. Both sweeps and outward interactions (Q4 and Q1) play significant roles in entrainment given their greater longitudinal velocities, many of which exceed the lifting thresholds. In contrast, the entrainment induced by ejections and inward interactions (Q2 and Q3) is dominated by rolling because of their inherently smaller longitudinal velocities $\left(u^{\prime}<0\right)$. The fractional contributions of Q2 and Q3 are, however, enhanced by the exposure effect associated with the well-sorted fine sediment mixture; such effect is exerted on those coarser grains with sizes $>D_{50}$. As a result, the proportions entrained by rolling are increased from 11.2 and $7.1 \%$ to 14.1 and $9.7 \%$ in Q2 and Q3, respectively. However, as the poorly sorted fine sediment mixture is used, the hiding effect exerted on those finer grains with sizes $<D_{50}$ is reduced, leading to the higher correction factors for effective lift forces (in average 13\% higher than the corresponding values of the well-sorted fine mixture). The elevated correction factors would in turn reduce the threshold velocities required for lifting. Once the lifting thresholds are lowered, the entrainment in all quadrants would be consistently dominated by lifting, such that the fractional contribution of Q2 (ejections) would exceed that of Q1 (outward interactions) since ejections are so much more common than outward interactions.

Under all kinds of coarse sediment mixtures, rolling is consistently the dominant mode of entrainment in four quadrants. The fractional contribution of Q2 (ejections) consistently exceeds that of Q1 (outward interactions) because ejections are much more common events. Although the time fraction of Q3 (inward interactions) is slightly greater than that of Q1 (outward interactions), the fractional contributions of Q3 are consistently the least significant ones due to the inherently smaller longitudinal velocities $\left(u^{\prime}<0\right)$. As the value of $\sigma_{g}$ becomes greater, the proportions entrained by lifting are increased in all quadrants. Under the poorly sorted coarse sediment mixture, the reduced hiding effect exerted on those finer grains leads to the higher correction factors for effective drag and lift forces (in average 16\% higher than the corresponding values of the well-sorted coarse mixture). The elevated correction factors would reduce the threshold velocities required for entrainment of those finer grains. The reduced threshold velocities in turn enhance the fractional contributions of Q2 and Q3, both associated with negative values of $u^{\prime}$. 


\section{Conclusions}

The role of turbulent bursting in sediment entrainment is numerically investigated in this study. The results reveal that the time fractions of four quadrants remain constant under typical values of bed-shear stress. Sweeps (Q4) are consistently the most significant contributor to sediment entrainment under various flow and sediment conditions. The fractional contributions of ejections and inward interactions (Q2 and Q3) increase with the standard deviation of grain-size distribution. Under the poorly sorted sediment mixtures, the fractional contribution of ejections (Q2) would exceed that of outward interaction (Q1). Experimental studies should be conducted to further verify these results. Although plausible, the simple mechanistic model adopted in this work may be revised in the future by incorporating the vertical velocity component. In addition, the higher-order correlations of near-bed $2 \mathrm{D}$ velocities under various conditions can be also derived from the experimental studies to better predict the turbulent bursting and sediment motions.

\section{Acknowledgments}

The writers acknowledge the research funding granted by the $\mathrm{Na}-$ tional Science Council, Taiwan, R.O.C. Comments from the ASCE reviewers helped improve the clarity of this work.

\section{Notation}

The following symbols are used in this technical note:

$$
\begin{aligned}
A= & \text { exposed frontal area; } \\
B_{L}, B_{R}= & \text { lifting and rolling thresholds; } \\
C_{D}, C_{L}= & \text { drag and lift coefficients; } \\
D_{i}, D_{k}= & \text { grain sizes of fraction } i \text { and downstream } \\
& \text { supporting particle; } \\
D_{50}, D_{84}= & \text { median and } 84 \text { th-percentile grain sizes; } \\
F_{D}, F_{L}= & \text { instantaneous effective drag and lift forces; } \\
\mathrm{FC}_{i}= & \text { fractional contribution of quadrant } i=\left(\mathrm{PR}_{i}\right. \\
& +\mathrm{PL}) / \mathrm{PT} ; \\
g_{3}(U, V)= & \text { third-order Gram-Charlier joint pdf; } \\
k_{s}= & \text { equivalent roughness height }=2 D_{50} ; \\
k_{s}^{+}= & \text {roughness Reynolds number }=u_{*} k_{s} / \nu ; \\
L_{D}, L_{L}, L_{W}= & \text { moment arms of } F_{D}, F_{L}, \text { and } W ; \\
M_{12}, M_{21}= & \text { diffusion factors; } \\
P_{L}, P_{R}= & \text { lifting and rolling probabilities; } \\
\mathrm{PL}, \mathrm{PR}, \mathrm{PT}= & \text { mean lifting, rolling, and total entrainment } \\
& \text { probabilities; } \\
R_{u}, R_{v}, R_{u v}= & R_{u v} V-U, R_{u v} U-V, \text { and correlation } \\
& \text { coefficient; } \\
S_{u}, S_{v}= & \text { skewness factors; } \\
T_{i}= & \text { time fraction of quadrant } i ; \\
U, V= & u^{\prime} / \sigma_{u} \text { and } v^{\prime} / \sigma_{v} ; \\
U_{L}, V_{L}= & \text { ranges of } U \text { and } V \text { meeting the constraints } \\
& \text { for lifting and GC joint pdf; } \\
U_{R}, V_{R}= & \text { ranges of } U \text { and } V \text { meeting the constraints } \\
& \text { for rolling and } \mathrm{GC} \text { joint pdf; }
\end{aligned}
$$

$$
\begin{aligned}
U_{L, i}, V_{L, i}= & \text { partial ranges of } U_{L} \text { and } V_{L} \text { in quadrant } i \\
U_{R, i}, V_{R, i}= & \text { partial ranges of } U_{R} \text { and } V_{R} \text { in quadrant } i \\
u^{\prime}, v^{\prime}= & \text { longitudinal and vertical velocity } \\
& \text { fluctuations; } \\
u_{b}, v_{b}, \bar{u}_{b}, \bar{v}_{b}= & \text { near-bed instantaneous and mean } \\
& \text { longitudinal and vertical velocities; } \\
u_{l}, u_{u}, v_{l}, v_{u}= & \bar{u}_{b}+u_{l}^{\prime}, \bar{u}_{b}+u_{u}^{\prime}, \bar{v}_{b}+v_{l}^{\prime}, \text { and } \bar{v}_{b}+v_{u}^{\prime} ; \\
u_{l}^{\prime}, u_{u}^{\prime}, v_{l}^{\prime}, v_{u}^{\prime}= & \text { lower and upper bounds of } u^{\prime} \text { and } v^{\prime} \text { for } \\
& \text { GC joint pdf; } \\
u_{*}= & \text { bed-shear velocity }=\sqrt{\tau_{0} / \rho} ; \\
W= & \text { submerged weight; } \\
\Delta_{j}, \Delta_{k}= & \text { exposure and friction heights; } \\
\gamma_{s}, \gamma= & \text { specific weights of sediment and water; } \\
\theta= & \text { Shields number based on } \\
& D_{50}=\tau_{0} /\left(\gamma_{s}-\gamma\right) D_{50} ; \\
v, \rho= & \text { kinematic viscosity and density of fluid; } \\
\xi_{i}= & \text { hiding-exposure correction factor for grain } \\
& \text { size } D_{i} ; \\
\sigma_{g}= & \text { geometric standard deviation= } D_{84} / D_{50} ; \\
\sigma_{u}, \sigma_{v}= & \text { standard deviations of longitudinal and } \\
& \text { vertical velocities; and } \\
\tau_{0}= & \text { bed-shear stress. }
\end{aligned}
$$

\section{References}

Cheng, N.-S., and Chiew, Y.-M. (1999). "Closure to Discussion of 'Pickup probability for sediment entrainment." J. Hydraul. Eng., 125(7), 789.

Fischer, P. F., Leaf, G. K., and Restrepo, J. M. (2002). "Forces on particles in oscillatory boundary layers." J. Fluid Mech., 468, 327-347.

Frenkiel, F. N., and Klebanoff, P. S. (1973). "Probability distributions and correlations in a turbulent boundary layer." Phys. Fluids, 16, 725737.

Nakagawa, H., and Nezu, I. (1977). "Prediction of the contributions to the Reynolds stress from bursting events in open-channel flows." J. Fluid Mech., 80, 99-128.

Nelson, J. M., Shreve, R. L., McLean, S. R., and Drake, T. G. (1995). "Role of near-bed turbulence structure in bed load transport and bed form mechanics." Water Resour. Res., 31(8), 2071-2086.

Nezu, I., and Nakagawa, H. (1993). Turbulence in open-channel flows, Balkema, Rotterdam, The Netherlands.

Papanicolaou, A. N., Diplas, P., Dancey, C. L., and Balakrishnan, M. (2001). "Surface roughness effects in near-bed turbulence: Implications to sediment entrainment." J. Eng. Mech., 127(3), 211-218.

Pope, S. B. (2000). Turbulent flows, Cambridge University Press, New York.

Schmeeckle, M. W., and Nelson, J. M. (2003). "Direct numerical simulation of bedload transport using a local, dynamic boundary condition." Sedimentology, 50, 279-301.

Williams, J. J., Thorne, P. D., and Heathershaw, A. D. (1989). "Measurements of turbulence in the benthic boundary layer over a gravel bed." Sedimentology, 36, 959-971.

Wu, F.-C., and Chou, Y.-J. (2003). "Rolling and lifting probabilities for sediment entrainment." J. Hydraul. Eng., 129(2), 110-119.

Wu, F.-C., and Yang, K.-H. (2004). "Entrainment probabilities of mixedsize sediment incorporating near-bed coherent flow structures." J. Hydraul. Eng., 130(12), 1187-1197. 